\title{
Renal Biopsy Diagnosis of IGA Nephropathy in Chin Refugees Living in the United States
}

\author{
N. Rush, M. P. Goheen, C. L. Phillips \\ Indiana University School of Medicine, Pathology, Indianapolis, USA
}

IgA nephropathy (IgAN) is a common diagnosis in patients undergoing renal biopsy for hematuria and proteinuria, but histopathology may vary widely among affected patients. Worldwide, IgAN is the most common form of primary glomerulonephritis and is a leading cause of end-stage renal failure in patients presenting for renal replacement therapy ${ }^{1}$. In the United States, prevalence rates of IgA nephropathy are only 2 to 10 percent while in Asia prevalence rates range from 20 to $40 \%{ }^{2}$. In Chin refugees relocated from Myanmar (formerly Burma) to a geographic area near our laboratory in the Midwestern United States (U.S.), IgAN was the most frequently encountered glomerulopathy detected in needle biopsy specimens. We report our biopsy experience with this small but unique cohort, many of whom have had little to no previous access to medical care.

Our electronic database of surgical pathology specimens was searched for kidney biopsy samples originating from Chin patients who were born in Myanmar and immigrated to the Midwest region of the U.S. Needle cores from renal biopsy specimens were examined by bright-field (BM), immunofluorescent (IF), and/or electron (EM) microscopy using conventional techniques. Available clinical history that accompanied each specimen was reviewed. When sufficient tissue was available, specimens were scored by BM using criteria developed by The International IgA Nephropathy Study Group (i.e., Oxford criteria) ${ }^{3}$.

Of 2790 renal biopsy specimens evaluated at our facility in the last six years, seven were obtained from Chin individuals who emigrated from Myanmar. All seven patients had been evaluated by a nephrologist. Six Chin patients were diagnosed with IgAN based on analysis by IF and EM: five females (ages 21 to 38 years) and one male (age 29 years). The $7^{\text {th }}$ Chin patient, a male (age 38 years), had podocyte foot process effacement detected by EM in the absence of immune deposits by IF and was diagnosed with minimal change disease (MCD). Female patients were more likely to be proteinuric (5/5), hematuric (4/5), hypertensive (4/5), and pregnant (4/5), with initial serum creatinine levels that ranged from 0.5 to $1.12 \mathrm{mg} / \mathrm{dL}$. Both males presented with hypertension, proteinuria, and acute renal failure. The patient with MCD had an initial creatinine of $1.2 \mathrm{mg} / \mathrm{dL}$. Applying Oxford histologic criteria for scoring IgAN, two patients had one indicator of less favorable prognosis, one patient had three indicators, one patient had no morphologic indicators, and two specimens had insufficient tissue for analysis.

A cohort of Myanmar's Chin minority has emigrated to the Midwestern U.S. where they presented with hypertension and abnormal urinalysis. Seven of these individuals underwent an ultrasound-guided renal biopsy procedure. Six were diagnosed with IgAN (Fig.1) and one with MCD (Fig. 2). While the majority of these patients had IgAN, their histomorphology and prognosis varied widely based on 
Oxford criteria. Given the greater likelihood of encountering IgAN in the Chin population, nephrologists may initially decide to defer or delay a renal biopsy procedure, but these clinicians would not have access to tissue-based Oxford scores that might alter clinical management.

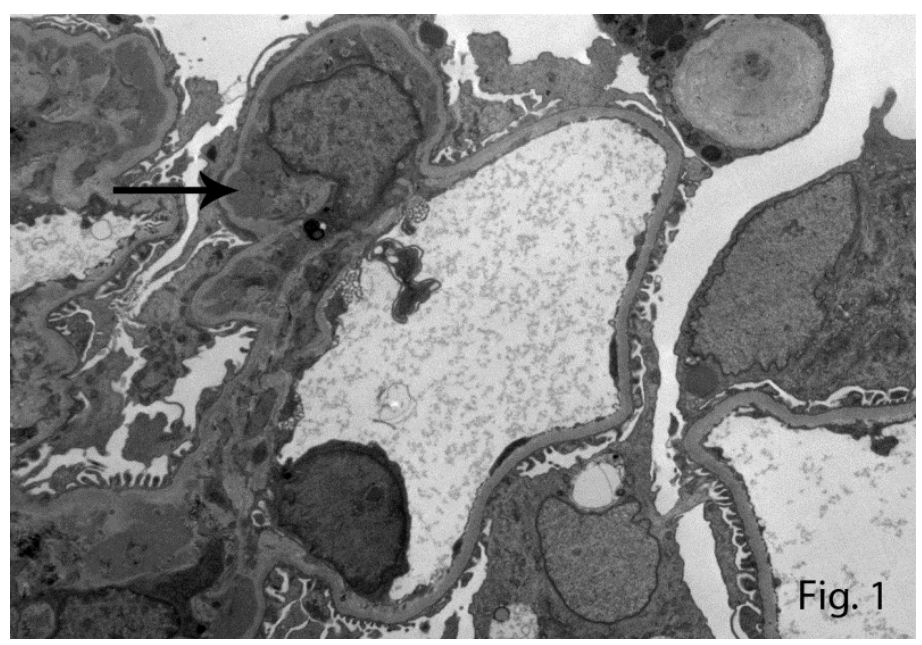

Fig.1 Immune deposit (arrow) in the mesangium of a patient with IgA nephropathy.

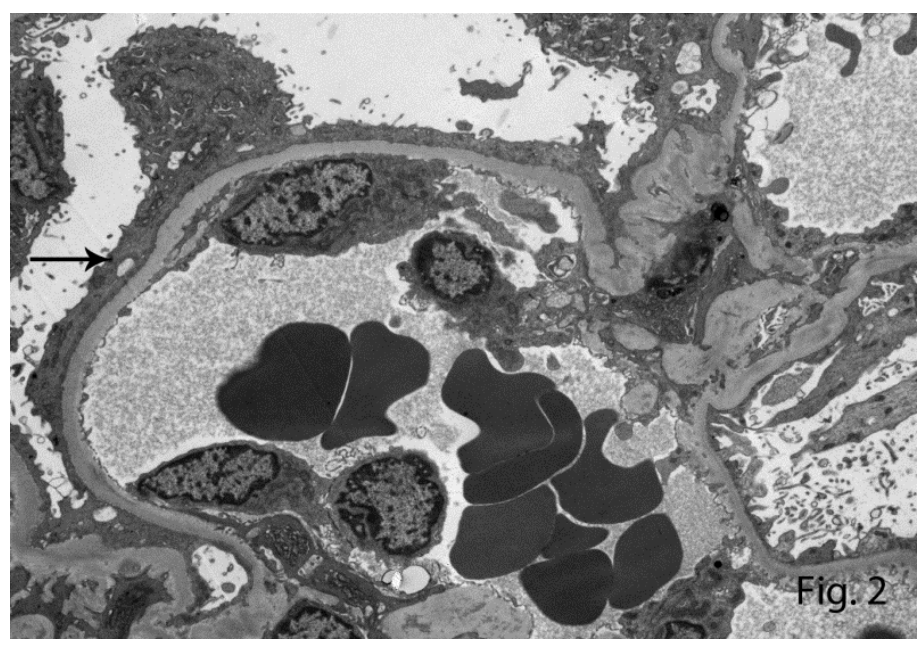

Fig. 2 Podocyte foot process effacement (arrow) in minimal change disease.

\section{References:}

1. Ibels LS, Györy AZ. Medicine (Baltimore). 1994 73(2):79-102.

2. Donadio JV, Grande JP.. N Engl J Med. 2002 347(10):738-48.

3. Coppo R, et al, The New Oxford Classification of IgA nephropathy 2010 (1) 241-8. 